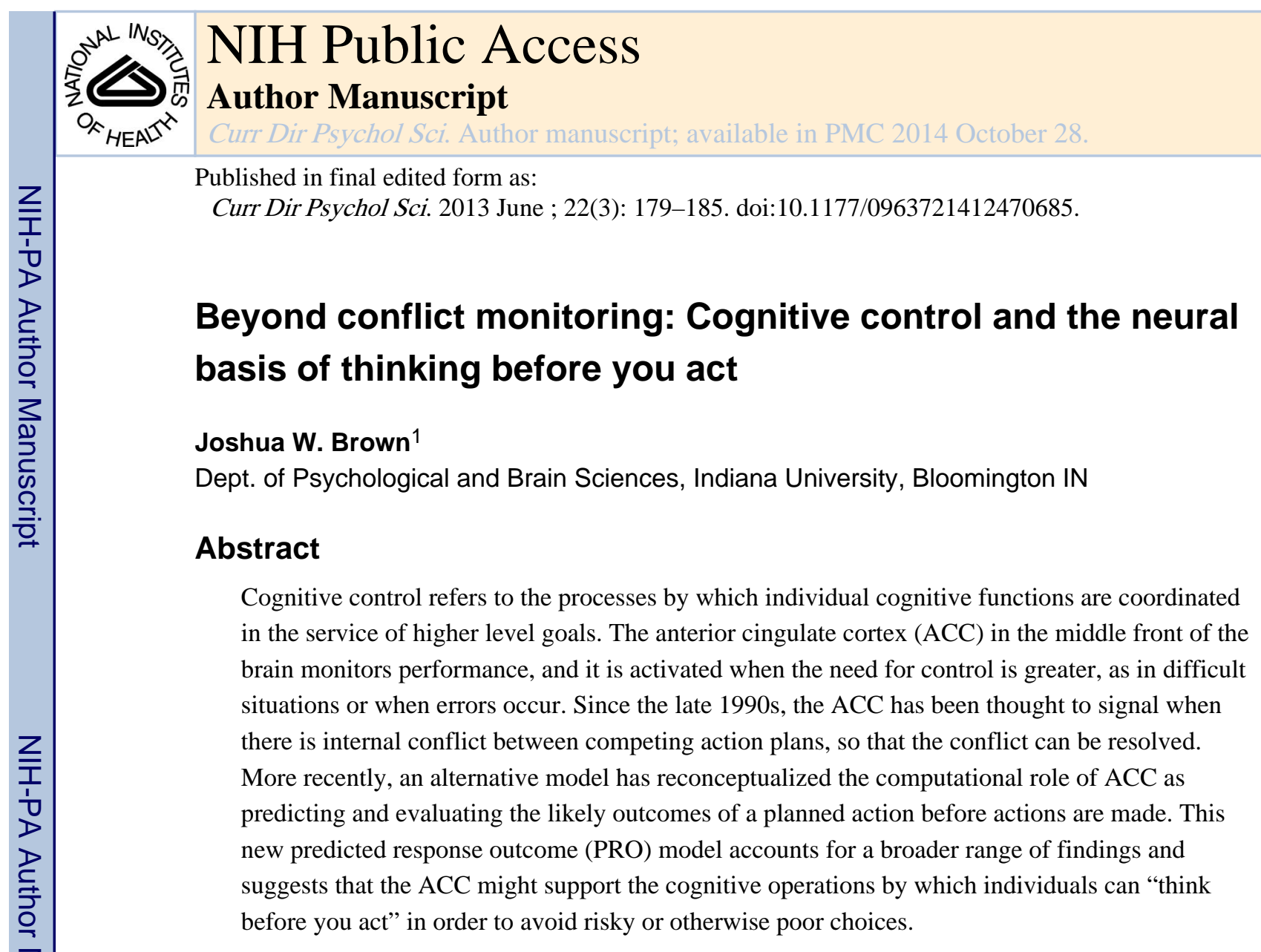

Keywords

cognitive control; performance monitoring; anterior cingulate cortex; risk; conflict monitoring

\title{
INTRODUCTION
}

Cognitive control (directing behavior toward goals) is heavily intertwined with performance monitoring, i.e. detecting when control must be exerted in the first place. Greater cognitive control is generally required in more difficult situations when automatic responses might otherwise lead to an error (Norman \& Shallice, 1986). Still, excessive cognitive control might waste limited cognitive resources. How then does an individual know when to exert greater control? The anterior cingulate cortex (ACC) emerged as a potential basis for performance monitoring beginning with the observation of error signals (Gehring, Coles, Meyer, \& Donchin, 1990). Interest in the ACC exploded with a series of papers proposing that ACC served to detect conflict between incompatible response processes (Botvinick, Braver, Barch, Carter, \& Cohen, 2001; Carter et al., 1998). For the last 15 years, the conflict monitoring model has become nearly synonymous with the ACC region, and the conflict model has inspired many studies of the ACC including our own.

Address correspondence to: Joshua W. Brown, Dept. of Psychological \& Brain Sciences, 1101 E Tenth St., Bloomington, IN 47405 USA, +1 812 855-9282 (office), +1 812 855-4691 (FAX), jwmbrown@indiana.edu, www.indiana.edu/ cclab. 
More recently though, a number of papers have either challenged the conflict monitoring hypothesis (Brown \& Braver, 2005; Holroyd \& Coles, 2002) or simply shown other effects in ACC that are not clearly accounted for by the conflict monitoring framework. These include error likelihood (Brown \& Braver, 2005, 2007), reward and unexpected reward (Ito, Stuphorn, Brown, \& Schall, 2003), non-stationarity in the environment (Behrens, Woolrich, Walton, \& Rushworth, 2007), positive correlations with reaction time (Grinband et al., 2011) (but see (Yeung, Cohen, \& Botvinick, 2011)), foraging behavior (Kolling, Behrens, Mars, \& Rushworth, 2012), and other effects. The number of effects has proliferated to the point that over $20 \%$ of a sample of thousands of published fMRI studies show some effects in the ACC (Yarkoni, Poldrack, Nichols, Van Essen, \& Wager, 2011).

\section{A NEW MODEL}

In Beckett's Waiting for Godot, two hapless characters wait endlessly for a character named Godot, who never arrives. It is easy enough to recognize an explicit signal that an event has occurred, but it is harder to build an expectation of an event, and then infer at a certain time that the event is not occurring, because if it were to occur, it would already have happened. Put simply, how and when does one determine that Godot isn't coming, or more generally, that reward is not to be found (Kolling et al., 2012)? Such an inference requires two pieces of information: First, the characters must form predictions about what will happen as a consequence of their waiting, and when it will happen. There is some probability that Godot will arrive by a certain time, and there is some probability that Godot will be a no-show, or perhaps that something else will interrupt the waiting period. Second, the characters must be able to compare their expectations about what will probably happen against what actually happens.

Here I argue that it is possible to account for the variety of ACC effects in a unified theoretical framework, which we call the Predicted Response Outcome (PRO) model (Alexander \& Brown, 2011). Like the characters in Waiting for Godot, the PRO model represents predictions about likely outcomes (Figure 1a,b), as well as separate evaluations of how well the predictions match the outcomes (Figure 1c,d). In this way, the new theory clarifies the psychological processes of how people predict the potential consequence of an action before acting, and how they evaluate the outcomes of their actions. A very similar computational framework has also been proposed independently by another group (Silvetti, Seurinck, \& Verguts, 2011). The model begins with an earlier proposal that ACC learns to predict the likelihood of various possible consequences of an action, especially errors (Brown \& Braver, 2005, 2007). When response conflict is present, errors are more likely, so conflict and error likelihood are often confounded (Brown \& Braver, 2005). These were deconfounded by isolating trials where the cue color indicated a higher error likelihood, but the conflicting stimulus nevertheless did not subsequently appear. The result was that ACC activity remained elevated when error likelihood was higher, even without response conflict (Brown \& Braver, 2005). Thus, apparent response conflict effects might reflect in part greater error likelihood rather than conflict. Further studies showed that loss-averse individuals had stronger ACC activity related to error likelihood, which suggested that ACC might not only predict potential errors but also control behavior to avoid errors (Brown \& Braver, 2007, 2008). 
Despite its successes, the error likelihood model was still incomplete. In the same neuroimaging study that showed error likelihood effects, we also found that ACC activity following an actual error (not just a predicted error) was higher when the error likelihood was lower (Brown \& Braver, 2005). The error likelihood computational model could not account for this effect of surprise. To address this, we returned to the idea of ACC as comparing actual vs. intended actions (or outcomes) (Holroyd \& Coles, 2002; Ito et al., 2003; Scheffers \& Coles, 2000), which had been a dominant account of ACC prior to the conflict model. The concept of an outcome predictor, as in the error likelihood model, and the concept of a comparator fit together remarkably well: the predictor (Figure 1a,b) simply provides the basis against which to compare the actual outcome (Figure 1e,f). This comparison is simply the difference between the expected and actual outcome (Ito et al., 2003), shown in Figure 1c,d. This structure is collectively the essence of the PRO model (Figure 1) (Alexander \& Brown, 2010, 2011; Silvetti et al., 2011).

A key function of the PRO model is detecting the absence of an expected outcome, for example that Godot did not come by the expected time. The model suggests that the ACC predicts not only error likelihood but more generally the respective likelihoods of all reasonably possible outcomes. Furthermore, the PRO model introduces a timing element: prediction-related activity increases over time and peaks when the outcome is expected to occur. Finally, the PRO model introduces the concept of negative surprise (Figure 1c,d). If the outcome does occur when predicted, then the outcome inhibits the prediction signal (Figure 1c,d). Conversely, if the outcome fails to occur at the expected time, then the prediction signal grows unabated. This unmet prediction signal, when it exceeds a certain threshold, indicates that a prediction error has occurred (Figure 1d). This is the essence of negative surprise, i.e. that an expected outcome failed to occur. Note that negative here does not necessarily mean aversive; an unexpected omission can be aversive (e.g. you expected reward but didn't get it) or appetitive (a police officer pulled you over and you expected a fine, but then he doesn't give you one).

Incidentally, the PRO model also computes positive surprise, in which predicted vs. actual outcomes are compared but with the signs reversed. The net result is a signal that detects events that occur unexpectedly, as distinct from negative surprise in which expected events fail to occur.

Are all of the prediction and negative surprise signals generated within the ACC, or do they originate from elsewhere in the brain? The ACC is a large and varied structure (Nee, Kastner, \& Brown, 2011), and conflict and error effects ascribed to ACC often extend into the pre-supplementary motor area (Rushworth, Hadland, Paus, \& Sipila, 2002). There is evidence that predictions of outcomes, apart from whether they are good or bad, are represented in the hippocampus (Van der Meer \& Redish, 2010) but integrated with desirability information in the ACC (Jahn, Nee, \& Brown, 2011). 


\section{MODEL ACCOUNTS OF EXISTING DATA}

\section{From conflict to foresight}

The dominant conflict model of ACC function achieved its status because it provided a simple and elegant account of the ACC. If the PRO model does not compute conflict, one might ask, how can it simulate conflict effects? The PRO model has been simulated as a computational neural model, and the basic computational properties of the model have been reported (Alexander \& Brown, 2010, 2011). Consider the case of an incongruent vs. congruent stimulus, as in the Flanker task where incongruent stimuli lead to ACC activation (Botvinick, Nystrom, Fissel, Carter, \& Cohen, 1999) (Figure 2). The congruent stimulus may reasonably lead to a predicted correct outcome, but the incongruent stimulus leads to a prediction of both a correct outcome and, with some probability, an incorrect outcome. The point is that incongruent stimuli predict two possible outcomes, but congruent stimuli predict a single outcome. When these activities are summed across a prediction layer (much as neural activity is aggregated in fMRI measures), the result is greater ACC activation for incongruent trials.

The PRO model makes a striking prediction that apparent conflict effects will not depend on actual conflict. In other words, if two possible outcomes are not mutually exclusive, then apparent conflict effects should still be found despite the absence of conflict. To test this, we asked subjects to perform a flanker task, pressing the left or right index finger buttons according to a central stimulus (Figure 2), with congruent or incongruent flankers. We then created an "ALL" condition, which removed the conflict: for incongruent stimuli, subjects were instructed that they should press both the left and right buttons simultaneously. The results showed that the same ACC regions signaling apparent conflict were also more active in the incongruent ALL condition, thus suggesting that apparent conflict effects do not depend on the presence of response conflict. The same results were found in a change signal task that also provides a response conflict manipulation, and we called this the "multiple response" effect (Brown, 2009).

\section{From foresight to better decisions}

It would be useful enough to have signals that predict the impending consequences of your actions, but it would be even more useful to predict the consequences before you execute an action. That way, you could avoid making poor choices in the first place. Could ACC provide such a signal that aversive consequences might follow? To test this, we asked subjects to choose between two buttons, one of which was usually rewarded, while the other was seldom rewarded. Then we asked subjects to simply imagine the consequences of each of the two possible actions in turn before making their selection. We found that when subjects contemplated the action that was less likely to pay off, there was more ACC activity, and this was distinct from anticipated response conflict or their subsequent choice (Jahn et al., 2011). This activity related to imagining an error would correspond to the Prediction component of the PRO model (i.e. predicting a probable error) rather than the Evaluation (negative surprise) component of the model. This finding also suggests that the ACC serves as a kind of early warning system, anticipating potential dangers so that they can be avoided. If so, then we might ask whether those who tend to avoid risky behavior 
show more ACC activity? The answer is yes: subjects with higher trait risk aversion show more sensitivity to error likelihood (Brown \& Braver, 2007). Furthermore, subjects who show more risk averse decisions in the Iowa Gambling Task and Balloon Analog Risk Task also show greater ACC activity during decision-making (Fukunaga, Brown, \& Bogg, 2012; Krawitz, Fukunaga, \& Brown, 2010).

\section{From error effects to surprise}

The first studies of the ACC described its sensitivity to errors (Gehring et al., 1990; Gemba, Sasaki, \& Brooks, 1986; Niki \& Watanabe, 1979), and error effects have been foundational to subsequent theories of ACC such as the conflict model. The PRO model makes a strong claim regarding error effects, namely that they do not reflect aversive outcomes per se. Instead, error effects are a specific case of the negative surprise discussed above. In many tasks that probe ACC error signals, error and unexpectedness are confounded, because error rates are typically under $10 \%$. What if error rates were very high (e.g. $90 \%$ or more)? In that case, an error would no longer be surprising. Would error effects persist? Or as the PRO model predicts, would they go away, or even invert themselves so that (surprisingly) correct trials led to greater ACC activity than (unsurprising) error trials? We tested this and found the latter to be the case, i.e. that ACC responds to surprise (Jessup, Busemeyer, \& Brown, 2010; Nee et al., 2011), as have others (Hayden et al., 2011; Oliveira, McDonald, \& Goodman, 2007; Wessel, Danielmeier, Morton, \& Ullsperger, 2012). Similarly, if an expected event occurs but at a surprising time, this also leads to surprise signals in the PRO model, a prediction that was supported by a subsequent fMRI study (Forster \& Brown, 2011).

\section{CURRENT DIRECTIONS}

The nature of ACC computations continues to be a topic of lively debate with significant implications, as the ACC is key to a number of clinical disorders including schizophrenia (Carter, MacDonald III, Ross, \& Stenger, 2001), drug abuse (Fishbein et al., 2005), and obsessive compulsive disorder (Fitzgerald et al., 2005). The PRO model suggests how these may play out as impairments of the ACC prediction mechanisms. For example, schizophrenia may be understood in part as involving a failure to accurately predict the likely outcomes of one's actions (Krawitz et al., 2011), while drug abuse may reflect an impaired ability to represent especially the risks associated with drug taking (Brown \& Braver, 2007, 2008). Conversely, the compulsions of OCD may reflect an excessive prediction of the risks associated with ongoing behavior (Fitzgerald et al., 2005).

Going forward, a number of questions remain open. The first is how exactly (and how strongly) the predictive and evaluative ACC signals as posited by the PRO model may influence decision-making (Fukunaga et al., 2012)? Relatedly, how do they influence current goals and working memory contents? A better understanding of these issues will be the key to a deeper theoretical understanding of the role of ACC dysfunction in clinical disorders. A second question is how the ACC avoidance signals relate to various definitions of risk - does ACC represent the probability of aversive outcomes? Or does it represent the variance of all possible outcomes? (Schonberg, Fox, \& Poldrack, 2011). A third question is 
how information flows in the cognitive control networks involving the ACC, and where the outcome predictions originate.

\section{Acknowledgments}

This research was supported in part by R01 DA026457 (JB), and the Indiana METACyt Initiative of Indiana University, funded in part through a major grant from the Lilly Endowment, Inc. Supported in part by the Intelligence Advanced Research Projects Activity (IARPA) via Department of the Interior (DOI) contract number D10PC20023. The U.S. Government is authorized to reproduce and distribute reprints for Governmental purposes notwithstanding any copyright annotation thereon. The views and conclusions contained herein are those of the authors and should not be interpreted as necessarily representing the official policies or endorsements, either expressed or implied, of IARPA, DOI or the U.S. Government.

\section{References}

Alexander WH, Brown JW. Computational models of performance monitoring and cognitive control. Topics in Cognitive Science. 2010; 2:658-677. [PubMed: 21359126]

Alexander WH, Brown JW. Medial prefrontal cortex as an action-outcome predictor. Nat Neurosci. 2011; 14(10):1338-1344. doi:10.1038/nn.2921. [PubMed: 21926982]

Behrens TE, Woolrich MW, Walton ME, Rushworth MF. Learning the value of information in an uncertain world. Nat Neurosci. 2007; 10(9):1214-1221. Retrieved from http:// www.ncbi.nlm.nih.gov/entrez/query.fcgi? cmd=Retrieve \&db=PubMed\&dopt=Citation\&list_uids=17676057. [PubMed: 17676057]

Botvinick MM, Braver TS, Barch DM, Carter CS, Cohen JC. Conflict monitoring and cognitive control. Psychological Review. 2001; 108:624-652. [PubMed: 11488380]

Botvinick MM, Nystrom L, Fissel K, Carter CS, Cohen JD. Conflict monitoring versus selection-foraction in anterior cingulate cortex. Nature. 1999; 402(6758):179-181. [PubMed: 10647008]

Brown JW. Conflict effects without conflict in anterior cingulate cortex: Multiple response effects and context specific representations. Neuroimage. 2009; 47(1):334-341. doi:10.1016/j.neuroimage. 2009.04.034. [PubMed: 19375509]

Brown JW, Braver TS. Learned Predictions of Error Likelihood in the Anterior Cingulate Cortex. Science. 2005; 307(5712):1118-1121. [PubMed: 15718473]

Bush G, Luu P, Posner MI. Cognitive and emotional influences in anterior cingulate cortex. Trends Cogn Sci. 2000; 4(6):215-222. Retrieved from http://www.biomednet.com/library/fulltext/ TICS.etd00345_13646613_v0004i06_00001483. [PubMed: 10827444]

Carter CS, Braver TS, Barch DM, Botvinick MM, Noll DC, Cohen JD. Anterior cingulate cortex, error detection, and the online monitoring of performance. Science. 1998; 280:747-749. [PubMed: 9563953]

Carter CS, MacDonald AW III, Ross LL, Stenger VA. Anterior cingulate cortex activity and impaired self-monitoring of performance in patients with schizophrenia: an event-related fMRI study. American Journal of Psychiatry. 2001; 158:1423-1428. [PubMed: 11532726]

Fishbein DH, Eldreth DL, Hyde C, Matochik JA, London ED, Contoreggi C, Kurian V, et al. Risky decision making and the anterior cingulate cortex in abstinent drug abusers and nonusers. Brain Res Cogn Brain Res. 2005; 23(1):119-136. Retrieved from http://www.ncbi.nlm.nih.gov/entrez/ query.fcgi? $\mathrm{cmd}=$ Retrieve $\& \mathrm{db}=$ PubMed\&dopt=Citation\&list_uids=15795139. [PubMed: 15795139]

Fitzgerald KD, Welsh RC, Gehring WJ, Abelson JL, Himle JA, Liberzon I, Taylor SF. Error-related hyperactivity of the anterior cingulate cortex in obsessive-compulsive disorder. Biol Psychiatry. 2005; 57(3):287-294. Retrieved from http://www.ncbi.nlm.nih.gov/entrez/query.fcgi? $\mathrm{cmd}=$ Retrieve $\& \mathrm{db}=$ PubMed\&dopt=Citation\&list_uids=15691530. [PubMed: 15691530]

Forster SE, Brown JW. Medial prefrontal cortex predicts and evaluates the timing of action outcomes. Neuroimage. 2011; 55(1):253-265. doi:10.1016/j.neuroimage.2010.11.035. [PubMed: 21094259]

Fukunaga R, Brown JW, Bogg T. Decision making in the Balloon Analogue Risk Task (BART): Anterior cingulate cortex signals loss aversion but not the infrequency of risky choices. Cognitive, affective \& behavioral neuroscience. 2012; 12(3):479-90. doi:10.3758/s13415-012-0102-1. 
Gehring WJ, Coles MGH, Meyer DE, Donchin E. The error-related negativity: An event-related potential accompanying errors. Psychophysiology. 1990; 27:S34.

Gemba H, Sasaki K, Brooks VB. "Error" potentials in limbic cortex (anterior cingulate area 24) of monkeys during motor learning. Neurosci Lett. 1986; 70(2):223-227. Retrieved from http:// www.ncbi.nlm.nih.gov/entrez/query.fcgi? $\mathrm{cmd}=$ Retrieve $\& \mathrm{db}=$ PubMed $\&$ dopt=Citation\&list_uids=3774229. [PubMed: 3774229$]$

Grinband J, Savitskaya J, Wager TD, Teichert T, Ferrera VP, Hirsch J. The dorsal medial frontal cortex is sensitive to time on task, not response conflict or error likelihood. Neuroimage. 2011; 57(2):303-311. doi:S1053-8119(10)01610-1 [pii] 10.1016/j.neuroimage.2010.12.027. [PubMed: 21168515]

Hayden BY, Heilbronner SR, Pearson JM, Platt ML. Surprise signals in anterior cingulate cortex: neuronal encoding of unsigned reward prediction errors driving adjustment in behavior. $\mathrm{J}$ Neurosci. 2011; 31(11):4178-4187. doi:31/11/4178 [pii] 10.1523/JNEUROSCI.4652-10.2011. [PubMed: 21411658]

Hollerman JR, Tremblay L, Schultz W. Influence of reward rexpectation on behavior-related neuronal activity in primate striatum. Journal of Neurophysiology. 1998; 80(2):947-963. [PubMed: 9705481]

Holroyd CB, Coles MG. The neural basis of human error processing: Reinforcement learning, dopamine, and the error-related negativity. Psych. Rev. 2002; 109(4):679-709. Retrieved from http://www.ncbi.nlm.nih.gov/entrez/query.fcgi? $\mathrm{cmd}=$ Retrieve $\& \mathrm{db}=$ PubMed\&dopt=Citation\&list_uids=12374324.

Ito S, Stuphorn V, Brown JW, Schall JD. Performance Monitoring by Anterior Cingulate Cortex During Saccade Countermanding. Science. 2003; 302:120-122. [PubMed: 14526085]

Jahn A, Nee DE, Brown JW. The neural basis of predicting the outcomes of imagined actions. Front Neurosci. 2011; 5:128. doi:10.3389/fnins.2011.00128. [PubMed: 22131965]

Jessup RK, Busemeyer JR, Brown JW. Error effects in anterior cingulate cortex reverse when error likelihood is high. J. Neurosci. 2010; 30(9):3467-3472. [PubMed: 20203206]

Kolling N, Behrens TEJ, Mars RB, Rushworth MFS. Neural Mechanisms of Foraging. Science. 2012; 336(6077):95-98. doi:10.1126/science.1216930. [PubMed: 22491854]

Krawitz A, Braver TS, Barch DM, Brown JW. Impaired error-likelihood prediction in medial prefrontal cortex in schizophrenia. Neuroimage. 2011; 54(2):1506-1517. doi:10.1016/ j.neuroimage.2010.09.027. [PubMed: 20851194]

Krawitz A, Fukunaga R, Brown JW. Anterior insula activity predicts the influence of positively framed messages on decision making. Cogn Affect Behav Neurosci. 2010; 10(3):392-405. doi: 10/3/392 [pii] 10.3758/CABN.10.3.392. [PubMed: 20805540]

Nee DE, Kastner S, Brown JW. Functional heterogeneity of conflict, error, task-switching, and unexpectedness effects within medial prefrontal cortex. Neuroimage. 2011; 54(1):528-540. doi:S1053-8119(10)01109-2 [pii] 10.1016/j.neuroimage.2010.08.027. [PubMed: 20728547]

Niki H, Watanabe M. Prefrontal and cingulate unit activity during timing behavior in the monkey. Brain Res. 1979; 171(2):213-224. Retrieved from http://www.ncbi.nlm.nih.gov/entrez/query.fcgi? $\mathrm{cmd}=$ Retrieve $\& \mathrm{db}=$ PubMed\&dopt=Citation\&list_uids=111772. [PubMed: 111772]

Norman, D.; Shallice, T. Attention to action: Willed and automatic control of behavior. In: Davidson, R.; Schwartz, G.; Shapiro, D., editors. Consciousness and Self Regulation: Advances in Research and Theory. Vol. 4. Plenum; New York: 1986.

Oliveira FT, McDonald JJ, Goodman D. Performance Monitoring in the Anterior Cingulate is Not All Error Related: Expectancy Deviation and the Representation of Action-Outcome Associations. J Cogn Neurosci. 2007 Retrieved from http://www.ncbi.nlm.nih.gov/entrez/query.fcgi? cmd=Retrieve $\& d b=$ PubMed\&dopt=Citation\&list_uids=17892382.

Rushworth MF, Hadland KA, Paus T, Sipila PK. Role of the human medial frontal cortex in task switching: a combined fMRI and TMS study. J Neurophysiol. 2002; 87(5):2577-2592. Retrieved from http://www.ncbi.nlm.nih.gov/entrez/query.fcgi? cmd=Retrieve \&db=PubMed\&dopt=Citation\&list_uids=11976394. [PubMed: 11976394]

Scheffers MK, Coles MG. Performance monitoring in a confusing world: error-related brain activity, judgments of response accuracy, and types of errors. J Exp Psychol Hum Percept Perform. 2000; 
26(1):141-151. Retrieved from http://www.ncbi.nlm.nih.gov/entrez/query.fcgi? $\mathrm{cmd}=$ Retrieve $\& \mathrm{db}=$ PubMed\&dopt=Citation\&list_uids=10696610. [PubMed: 10696610]

Schonberg T, Fox CR, Poldrack RA. Mind the gap: bridging economic and naturalistic risk-taking with cognitive neuroscience. Trends Cogn Sci. 2011; 15(1):11-19. doi:S1364-6613(10)00236-6 [pii] 10.1016/j.tics.2010.10.002. [PubMed: 21130018]

Silvetti M, Seurinck R, Verguts T. Value and prediction error in medial frontal cortex: integrating the single-unit and systems levels of analysis. Front Hum Neurosci. 2011; 5:75. doi:10.3389/fnhum. 2011.00075. [PubMed: 21886616]

Van der Meer MA, Redish AD. Expectancies in decision making, reinforcement learning, and ventral striatum. Frontiers in Neuroscience. 2010; 4(1):29-37. doi:10.3389/neuro.01.006.2010.

Wessel JR, Danielmeier C, Morton JB, Ullsperger M. Surprise and Error: Common Neuronal Architecture for the Processing of Errors and Novelty. Journal of Neuroscience. 2012; 32(22): 7528-7537. doi:10.1523/JNEUROSCI.6352-11.2012. [PubMed: 22649231]

Yarkoni T, Poldrack RA, Nichols TE, Van Essen DC, Wager TD. Large-scale automated synthesis of human functional neuroimaging data. Nature methods. 2011; 8(8):665-70. doi:10.1038/nmeth. 1635. [PubMed: 21706013]

Yeung N, Cohen JD, Botvinick MM, et al. Errors of interpretation and modeling: A reply to Grinband. Neuroimage. 2011; 57(2):316-319. doi:S1053-8119(11)00428-9 [pii] 10.1016/j.neuroimage. 2011.04.029. [PubMed: 21530662] 


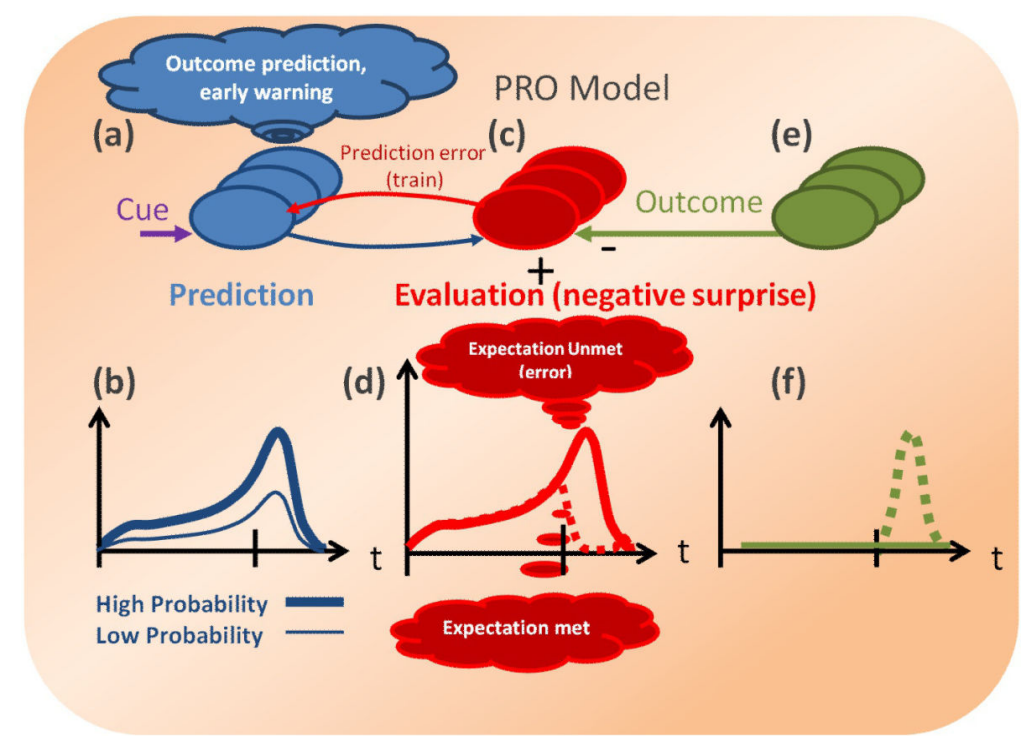

Figure 1.

The Predicted Response Outcome (PRO) model of anterior cingulate cortex. (a) The Prediction units generate a timed prediction of what outcomes are expected, with what probability, and when. (b) Timecourse of Prediction units. Greater probabilities are associated with greater prediction activity, and the activity peaks at the time when the outcome is expected. Prediction signals of aversive outcomes might influence decisions away from risk. (c) The Evaluation units compute negative surprise, i.e. they detect when an expected outcome fails to occur. This is computed simply by subtracting the actual outcome signal off (via inhibition) from the Prediction unit signal. (d) Timecourse of Evaluation unit activity. Events that are predicted with a high probability yield greater surprise signals when they fail to occur (solid line), relative to when they do occur as expected (dashed line). This mechanism accounts for error signals within ACC and might drive corrective actions when errors occur. (e) Outcome units signal when an actual outcome occurs. (f) An Outcome unit is transiently activated at the moment when a corresponding outcome actually occurs (dashed line), or if no outcome occurs, the Outcome unit remains inactive (solid line). 


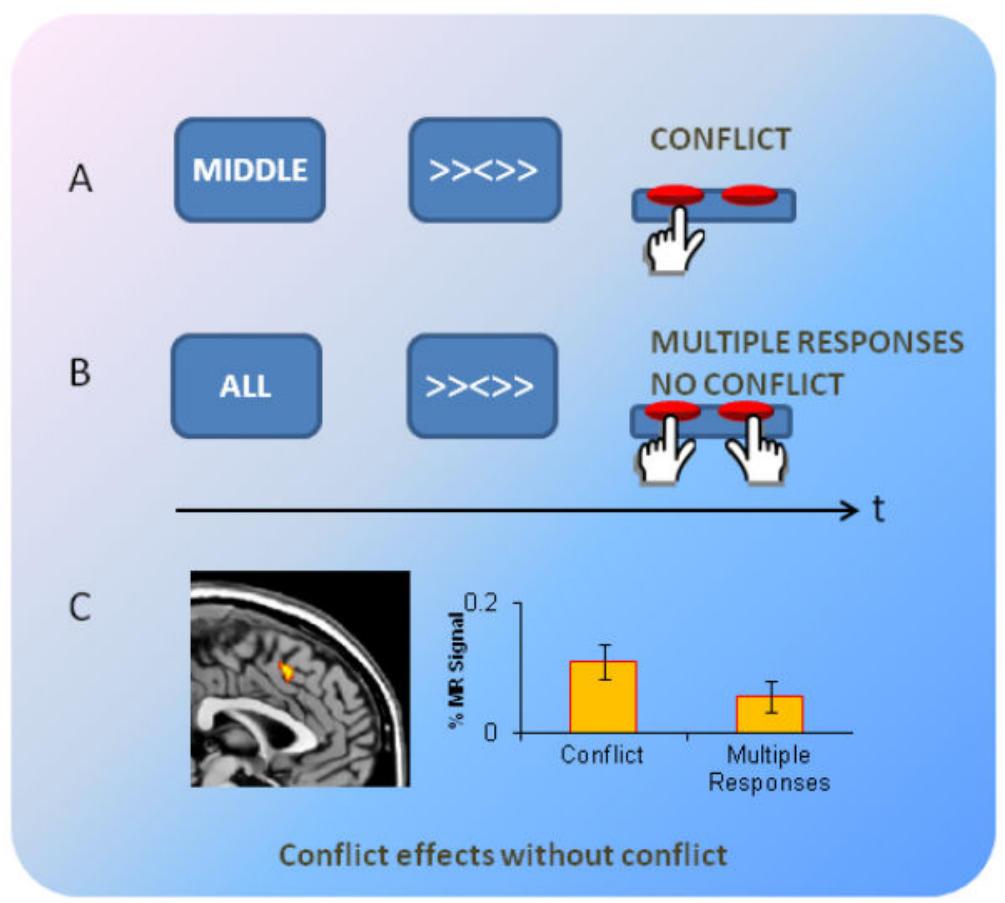

Figure 2.

Conflict effects without conflict computation as predicted by the PRO model. (A) In the standard flanker task instructed here by the "MIDDLE" cue, subjects must press a button corresponding to the direction of the arrow in the middle and ignore the flankers. Here, the middle arrow points to the left, and so the subject presses only the left button. (B) In the modified flanker task instructed here by the "ALL" cue, subjects must press one or both buttons as cued by both the middle and the flanker arrows. Here, the middle and flanker arrows point in both directions, but the subject need not suppress the response to the incongruent flankers. So the subject presses both buttons simultaneously. (C) The dorsal ACC shows greater activity for the incongruent relative to the congruent conditions, in both the "middle" and "all" conditions, suggesting that apparent response conflict effects in ACC may not depend on conflict. Adapted from Brown, 2009. 